\title{
Feasibility study on magnetic enhanced flocculation for mitigating membrane fouling
}

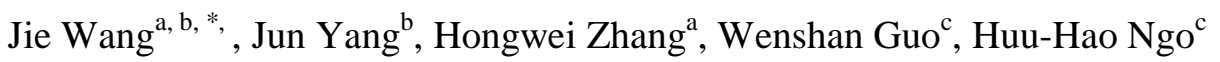 \\ ${ }^{\text {a }}$ State Key Laboratory of Hollow Fiber Membrane Materials and Processes, Tianjin Polytechnic University, \\ Tianjin 300387, China \\ ${ }^{\mathrm{b}}$ School of Environmental and Chemical Engineering, Tianjin Polytechnic University, Tianjin 300387, China \\ ${ }^{c}$ Centre for Technology in Water and Wastewater, School of Civil and Environmental Engineering, University \\ of Technology Sydney, NSW 2007, Australia
}

\footnotetext{
" Corresponding author at: State Key Laboratory of Hollow Fiber Membrane Materials and Processes \& School of Environmental and Chemical Engineering, Tianjin Polytechnic University, Tianjin 300387, China.

Tel.: +862283955673.

E-mail address: wangjie @tjpu.edu.cn (J. Wang)
}

\begin{abstract}
During coagulation/flocculation-membrane filtration (CF-MF) process, membrane fouling was alleviated more significantly through magnetic enhanced flocculation-membrane filtration (MEF-MF) in the presence of ferromagnetic seeds in coagulants. Porous cake layer with flocs of large size was able to alleviate decline rate of membrane flux. Foulant analysis proved that magnetic enhanced flocculation (MEF) pretreatment was more efficient for the reductions of low and mid-molecular weight (MW) organic structures than CF-MF. Biopolymers with high molecular weight were also effectively removed before filtration. Overall, MEF-MF could provide a novel alternative approach to mitigate membrane fouling for surface water treatment.
\end{abstract}

Keywords: drinking water treatment; membrane filtration; magnetic enhance flocculation; membrane fouling

Abbreviations: MEF-MF, magnetic enhanced flocculation membrane filtration; CFMF,coagulation/flocculation membrane filtration; MEF, magnetic enhanced flocculation; 
TMP, transmembrane pressure; DOM, dissolved organic matter; COD, chemical oxygen demand; SS, suspended solid; BSA, bovine serum albumin; DOC, dissolved organic carbon; ZP, zeta potential; PVDF, polyvinylidene fluoride; FC, ferric chloride; EEM, fluorescence excitation emission matrix; GPC, gel permeation chromatography; MWD, molecular weight distribution; FTIR, Fourier transform infrared spectroscopy; $D_{f}$, fractal dimension

\section{Introduction}

Membrane fouling is the major constraint in the implementation of membrane processes for drinking water treatment [1], as fouling increases operational costs, reduces permeate production and/or increases transmembrane pressure (TMP) [2] and [3]. Researches showed that pretreatment of surface water was very important to alleviate membrane fouling [4] and [5]. Although there were evidences in the literature to demonstrate that conventional flocculation could remove colloidal and dissolved organic matter (DOM) during microfiltration [6] and [7], significant membrane fouling was still observed according to seasonal conditions with pre-flocculation [8].

Enhanced flocculation pretreatment is one of the efficient techniques for mitigating membrane fouling [7]. It was found that non-reactive chemical additives such as zeolite, chitosan, activated carbon and cationic polymers were applied in pretreatment to reduce the concentrations of foulants in raw water, so as to mitigate membrane fouling [9]. Leo et al. [10] reported that embedded zeolite reduced the fouling by humic acid initiated pore blocking. About $80 \%$ permeate flux of membrane was maintained during the filtration of humic acid solution. Lee et al. [11] observed that coagulation using chitosan could remove chlorella vulgaris effectively, which was helpful for membrane fouling reduction. Moreover, pretreatment by coagulation with powdered activated carbon before membrane filtration could form larger and more porous flocs than those formed during conventional coagulation [12]. Overall, the effect on membrane fouling mitigation was achieved by the adsorption of non-reactive chemical additives.

Ferromagnetic seeds enhanced flocculation can rapidly separate compounds from mixtures with high efficiency and minimal initial investment by the magnetic characteristic. The application of magnetic seeding flocculation enhances the collision efficiency and collision frequency of colloidal particles, as well as makes colloidal particles to aggregate into larger 
flocs due to the decrease of colloidal stability [13]. Thus, the magnetic enhanced flocculation was been applied in wastewater treatment to remove foulants [13], [14], [15], [16] and [17].

It was found that MEF was efficient to remove COD (94\%) and SS (71\%) in treating mine water with high turbidity [18]. Liu et al. [19] reported that magnetic-coagulation separation could rapidly and effectively remove algae, chlorophyll-a and other foulants from freshwater. Semblante also applied porous micro-sized magnetite to achieve a maximum adsorption of $5.18 \mathrm{mg} / \mathrm{g}$ bovine serum albumin (BSA) and successfully inhibited the protein-induced fouling of a commercial polyvinylidene fluoride (PVDF) membrane [20]. In addition, magnetic nanoparticles in inorganic coagulants and their coagulation performances were studied by Zhang [21]. The performance of magnetic poly-aluminum chloride of basicity 2.0 (MPACl2.0) was better than that of PAC on turbidity and DOC removals. Moreover, large, loose and weak flocs were produced by MPAC12.0, which were preferable to recycle magnetic nanoparticles.

To remove COD, SS, and turbidity, which are main constituents of membrane foulants, MEF process was first designed and applied to mitigate membrane fouling in the ultrafiltration for drinking water treatment. In the study, the performance of PVDF hollow fiber membrane with the addition of magnetic enhanced flocculation was examined for treating surface water. The mechanisms of MEF on mitigating membrane were investigated from the perspective of microcosmic morphology. Furthermore, the characteristics and formation of flocs were investigated to analysis the performance of cake layer and membrane fouling mitigation.

\section{Materials and methods}

\section{Characteristics of natural surface water}

The raw water was collected from Luan River in Tianjin, China. The characteristics of the surface water are presented in Table 1. 
Table 1. The characteristics of natural surface water.

\begin{tabular}{lll}
\hline Parameter & Unit & Value \\
\hline $\mathrm{pH}$ & - & $7.25 \pm 0.53$ \\
$\mathrm{UV}_{254}(\mathrm{abs})$ & $\mathrm{cm}^{-1}$ & $0.074 \pm 0.008$ \\
$\mathrm{TOC}$ & $\mathrm{mg} / \mathrm{L}$ & $8.05 \pm 1.78$ \\
$\mathrm{DOC}$ & $\mathrm{mg} / \mathrm{L}$ & $6.65 \pm 0.38$ \\
$\mathrm{TSS}$ & $\mathrm{mg} / \mathrm{L}$ & $3.85 \pm 0.45$ \\
Zeta potential & $\mathrm{mV}$ & $-30.5 \pm 0.97$ \\
Turbidity & $\mathrm{NTU}$ & $3.64 \pm 0.44$ \\
Temperature & ${ }^{\circ} \mathrm{C}$ & $18 \pm 3$ \\
$\mathrm{Fe}^{3+}$ & $\mathrm{mg} / \mathrm{L}$ & $0.57 \pm 0.05$ \\
\hline
\end{tabular}

\section{Experimental apparatus and preparation}

The bench-scale experimental setup is shown in Fig. 1. The system consists of a coagulant solution tank, a feeding tank, a membrane reactor and a permeate tank. Coagulant solution was pumped into the membrane reactor together with raw water. Colloidal particles were destabilized and furled in flocs with blending. The membrane module submerged in the mixture, and dead-end filtration was carried out for study membrane fouling phenomenon.

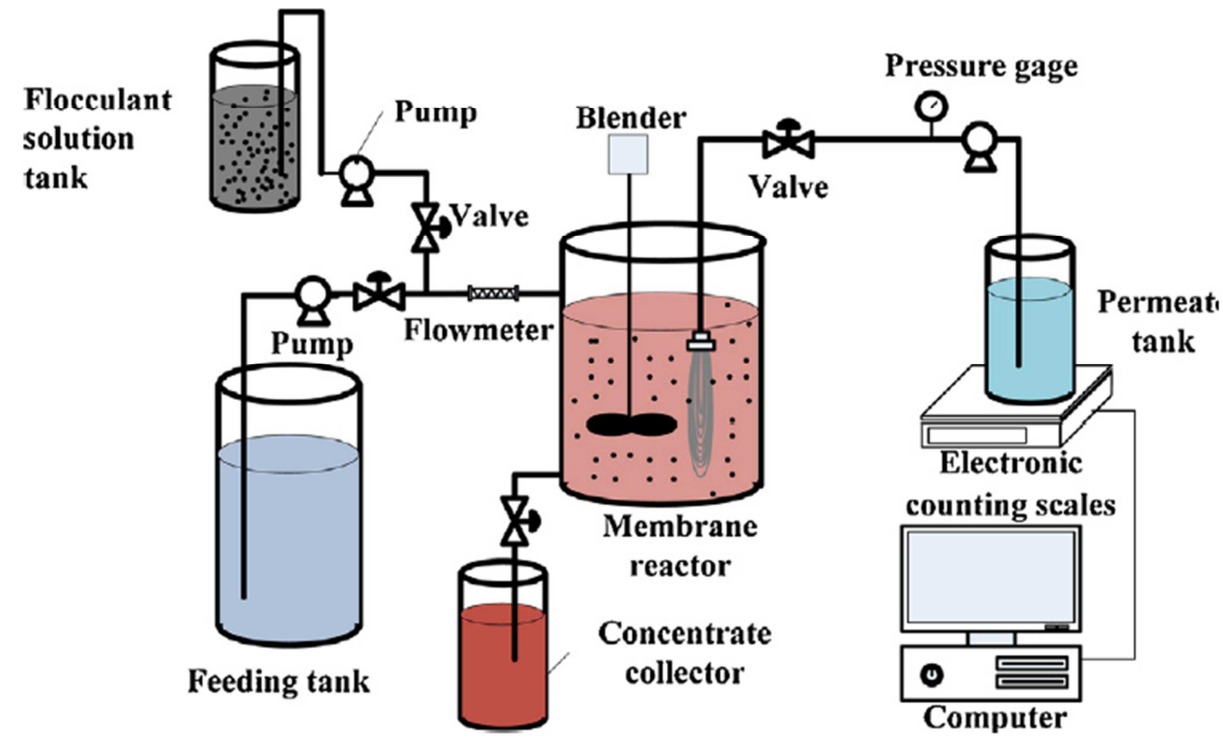

Fig. 1. Schematic diagram of the experimental setup.

Ferromagnetic seeds $\left(\mathrm{Fe}_{3} \mathrm{O}_{4}\right)$ (Kermel, Tianjin, China) with sizes from 20 to $60 \mu \mathrm{m}$ (refer to Fig. 2) was magnetized in a beaker for $5 \mathrm{~min}$ by a permanent magnet ( $40 \mathrm{mT}$ ) put under the beaker. The magnetic induction intensity of those ferromagnetic seeds was $0.01 \mathrm{mT}$. A novel coagulant was prepared by mixing ferromagnetic seeds in ferric chloride (FC) (Kermel, 
Tianjin, China) solution. The mass rate of ferromagnetic seeds and FC was 1:4. The novel coagulant with ferromagnetic seeds mixed in was a heterogeneous substance and should be shaken well in order to disperse as uniformly as possible. In this study, FC without any ferromagnetic seeds was adopted for contrast experiments.

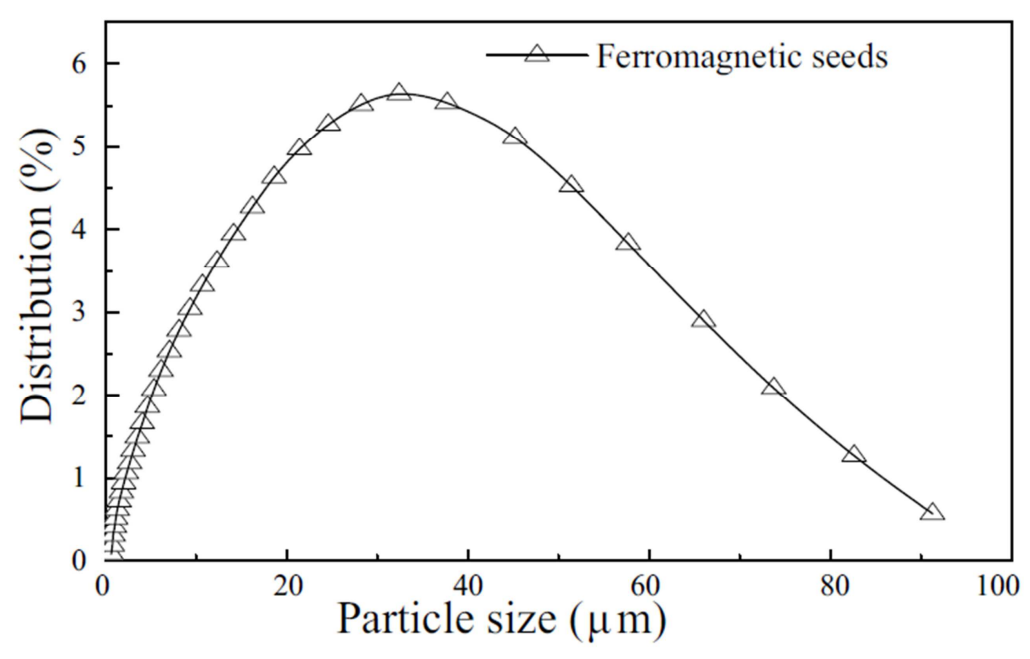

Fig. 2. The particle size distribution of ferromagnetic seeds.

For testing the effects of coagulants, a virgin PVDF hollow fiber ultrafiltration membrane module (MOTIMO Membrane Technology, Tianjin, China) was used in each experiment. The effective surface area and pore size of each module were $0.04 \mathrm{~m}^{2}$ and $0.1 \mu \mathrm{m}$, respectively. The operating pressure was remained constant over the filtration period. The module was immersed in deionized water for $24 \mathrm{~h}$ before use. After each experimental cycle, the fouled module was soaked in sodium hypochlorite (Kermel, Tianjin, China) solution (500 mg/L as free chlorine) for $10 \mathrm{~min}$ and rinsed with deionized water. The permeate tank was used to collect the effluent from the membrane reactor. The tank was placed on an electronic counting scale to measure the mass of permeate and the data were recorded by the computer every $10 \mathrm{~min}$.

For each experiment, the virgin membrane module was installed in the membrane reactor configured with an outside-in pressurization and low-intensity magnetic field. First, membrane module was pre-compacted by filtering deionized water for $1 \mathrm{~h}$ at constant pressure $(20 \mathrm{kPa})$ and temperature $\left(18 \pm 3{ }^{\circ} \mathrm{C}\right)$ until a constant permeate flux was achieved. After the pre-compaction, the membrane filtration system was stopped and deionized water was drained from the membrane reactor. Then, raw water mixed with coagulant was pumped 
into the membrane reactor continuously. After that, the membrane system was restarted and operated for $6 \mathrm{~h}$ under the same operating conditions as those in the pre-compaction run.

\section{Analysis of iron ion concentrations}

The concentrations of iron ion in the two coagulants and permeates were investigated. The concentrations of iron ion for each coagulants solution and permeate are listed inTable 2. The results showed that values obtained in the experiment were well consistent with those of dosage. The addition of ferromagnetic seeds didn't increase the concentration of iron ion for both of coagulants. Thus, the ferromagnetic seeds were quite stable in coagulants solution and flocculation solution.

Table 2. The concentration of $\mathrm{Fe}^{3+}(\mathrm{mg} / \mathrm{L})$ in surface water, coagulant solution $(20 \mathrm{mg} / \mathrm{L})$ and permeate.

\begin{tabular}{cccccc}
\hline \multirow{2}{*}{ Coagulant } & Unit & \multicolumn{2}{c}{ Coagulant solution } & Measured \\
values & $\begin{array}{c}\text { Theoretical } \\
\text { values }\end{array}$ & Permeate & $\begin{array}{c}\text { Surface } \\
\text { water }\end{array}$ \\
$\begin{array}{c}\text { Ferromagnetic } \\
\text { seeding mixed FC } \\
\text { FC }\end{array}$ & $\mathrm{mg} / \mathrm{L}$ & $5.54 \pm 0.073$ & 5.51 & $0.15 \pm 0.042$ & $0.57 \pm 0.059$ \\
\hline & $\mathrm{mg} / \mathrm{L}$ & $6.91 \pm 0.068$ & 6.89 & $0.2 \pm 0.048$ & \\
\hline
\end{tabular}

\section{Analytical methods}

Dissolved organic carbon of water samples, turbidity, $\mathrm{UV}_{254}$ absorbance, concentration of iron ion $\left(\mathrm{Fe}^{3+}\right)$ and the magnetic field intensity were measured using a combustion-type organic carbon analyzer (TOC-Vcph analyzer, Shimadzu, Kyoto, Japan), a turbidimeter (2100N, Hach, Colorado, USA), an ultraviolet spectrophotometer (T6, PERSEE, Beijing, China), inductive coupled plasma emission spectrometer (ICP) (715-ES, Varian, California, USA), and teslameter (HT20, Shanghai, China), respectively.

Molecular weight distribution of dissolved organic matter in water samples was determined by liquid chromatography (Waters 2695, Waters, Massachusetts, USA) with differential refractive index detectors (Waters 2414, Waters, Massachusetts, USA). The detectors had three size exclusion chromatography columns in order to separate organic molecules according to their molecular size. The columns used were Ultrahydrogel ${ }^{\mathrm{TM}} 500,200,120$ arranged in series (TSK-GEL, Waters, Massachusetts, USA) and were able to detect a wider range in molecular weight (150-400 thousand $\mathrm{Da})$. The column temperature, detector temperature and the mobile phase, water flow rate were kept at $55{ }^{\circ} \mathrm{C}, 50{ }^{\circ} \mathrm{C}$ and $0.6 \mathrm{~mL} / \mathrm{min}$, 
respectively. Sodium polystyrene sulfonates and polyethylene glycols were used as molecular weight calibration standards.

The particle size of ferromagnetic seeds and floc size were measured by laser particle analyzer (Mastersize2000, Malvern, Worcestershire, UK). The surface morphological features of the virgin and fouled membranes were investigated using a scanning electron microscope (SEM) (S-4800, Hitachi, Tokyo, Japan).The functional groups of foulants were analyzed by Fourier transform infrared spectroscopy (FTIR) (NICOLET6700, Thermo Fisher, Massachusetts, USA).

The fluorescence excitation emission matrix (EEM) spectroscopy of raw water and permeates of MEF-MF and CF-MF were detected by fluorescence spectrophotometer (F-7000, Hitachi, Tokyo, Japan). Prior to analysis, samples were filtered through a microfiltration membrane $(0.45 \mu \mathrm{m})$ for removing all the insoluble organic particles.

Zeta potential of solution and diameter of colloid particle were determined by a Malvern Instruments (Zetasizer Nano, Malvern, Worcestershire, UK). Surface ZP (zeta potential) was characterized by streaming potential [22] and [23]. The streaming potential across the membrane was measured with a pair of commercial $\mathrm{Ag} / \mathrm{AgCl}$ reference electrodes placed on the permeate sides and retentate sides close to the membrane module under the pressure gradients from 10 to $70 \mathrm{kPa}$. The measurement was performed with a $\mathrm{KCl}$ electrolyte at $1 \times 10^{-3} \mathrm{~mol} / \mathrm{L}$ at the $\mathrm{pH}$ of 7.2. Before the measurements of zetal potential, the electrostatic charges were stabilized for $1 \mathrm{~h}$ by flowing the $\mathrm{KCl}$ electrolyte solution through the membrane. The potential difference between the electrodes was measured and displayed on an oscilloscope (54641A, Agilent, California, USA). The surface zeta potential was then calculated by the Helmoltz-Smoluchowski principle.

\section{Modeling development}

In order to characterize membrane fouling, a simple membrane fouling index normalized flux $\left(F_{N}\right)$ was used, which is generally defined as follows:

$F_{N}=\frac{J_{S}}{J_{S 0}}$

where $J_{S 0}$ in Eq. (1) is the constant permeate flux $\left(\mathrm{L} /\left(\mathrm{m}^{2} \mathrm{~h}\right)\right)$ obtained from filtering deionized water; $J_{S}$ is gained flux by filtering the raw water after flocculation pretreatment. The normalized flux curve can reflect the tendency of membrane fouling with time. 
Fractal dimension was applied to character flocs properties [24] and [25], which can be calculated using the following equation:

$\mathrm{A}=\alpha \mathrm{LD}_{\mathrm{f}}$

where $A$ is the projection area of floc $\left(\mathrm{m}^{2}\right) ; L$ is maximum length of projection $(\mathrm{m}) ; \alpha$ is proportionality constant, $D_{f}$ is the fractal dimension of flocs in $2 \mathrm{D}$ space. The bigger fractal dimension of flocs the better effect of flocculation performs.

\section{Results and discussions}

\section{Membrane filtration performance}

\section{Membrane permeate flux decline}

The flux decline curves for CF-MF and MEF-MF processes over the entire filtration period are shown in Fig. 3. Both of the processes were operated for $6 \mathrm{~h}$ at $0.02 \mathrm{MPa}$ with the coagulants of FC and ferromagnetic seeds mixed FC at $20 \mathrm{mg} / \mathrm{L}$, respectively. The filtration curves of raw water and ferromagnetic seeds were also plotted to provide good baselines on flux performance.

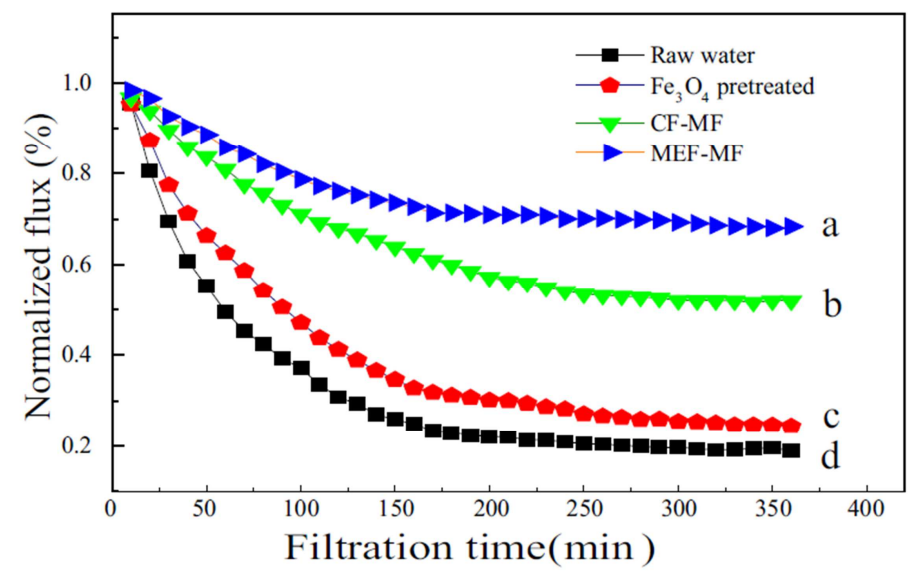

Fig. 3. Normalized flux variation through time with different coagulants. The raw water was pretreated by (a) MEF, (b) flocculation, (c) ferromagnetic seeds and (d) nothing, respectively. In each pretreatment the dosage of coagulant was $20 \mathrm{mg} / \mathrm{L}$.

As can be seen from Fig. 3, the membrane flux in the process of raw water direct ultrafiltration without any pretreatment presented a gradual decline rate after a dramatic decline in the initial filtration stage. The similar phenomenon of membranes flux decline rate also appeared in the ferromagnetic seeds pretreatment process. Whereas, the flux decline of the MEF pretreatment was relatively moderate over the filtration time. The fluxes of the raw 
water and ferromagnetic seeds decreased about $85 \%$ and $80 \%$ after 360 min filtration, respectively. However, the flux declined less than $50 \%$ when the ferromagnetic seeds were mixed together with coagulant. It was apparent that ferromagnetic seeds could not sustain the flux effectively without combining with coagulant. Therefore, FC mixed with ferromagnetic seeds as coagulant was more effective on mitigating fouling than using FC alone. 
To evaluate fouling reduction of MEF for in-pore and surface fouling formed during ultrafiltration of river water, cross-sectional SEM images were taken for the hollow fiber PVDF membrane samples before and after the filtration, as shown in Fig. 4.

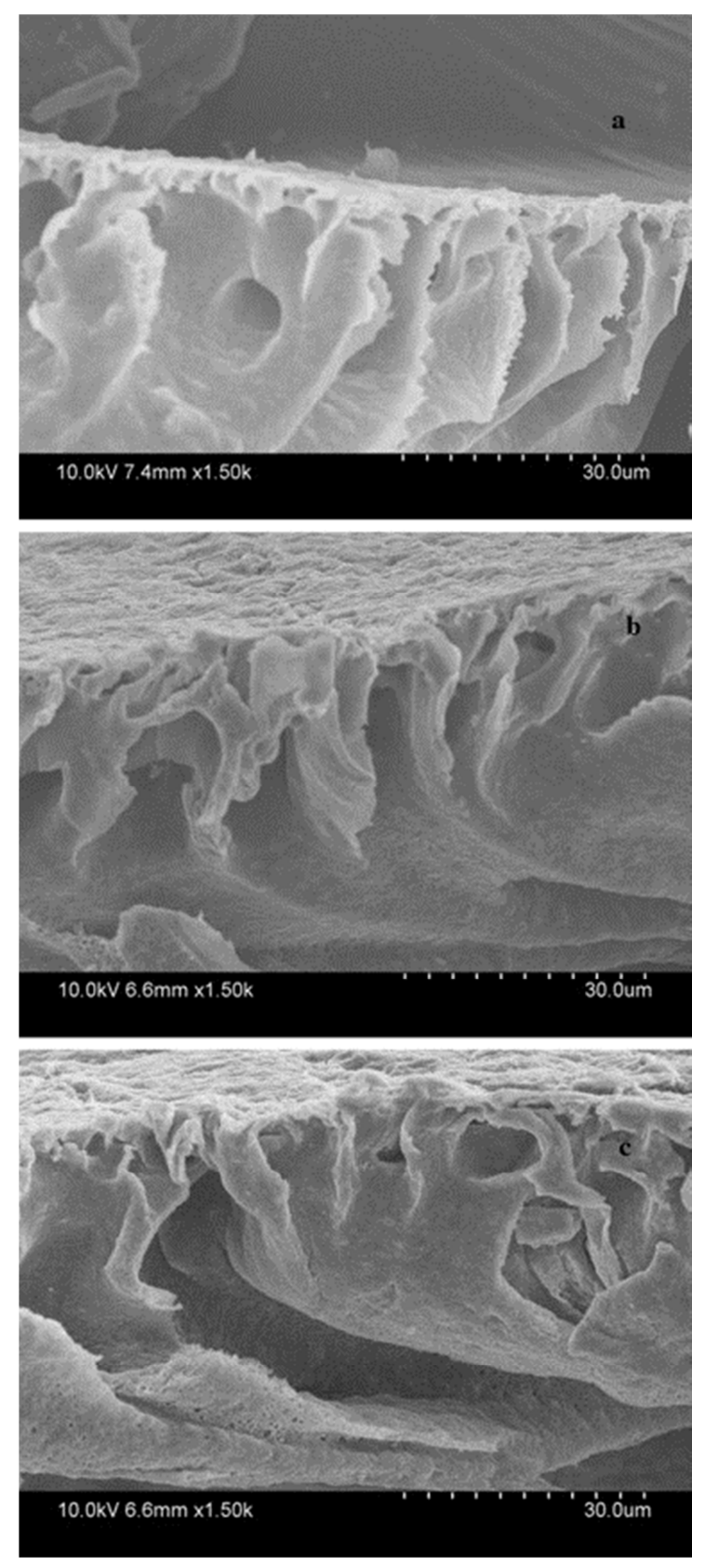

Fig. 4. SEM observation for the cross-section of the membrane. (a) The new membrane, (b) fouled membrane of MEF-MF and (c) fouled membrane of CF-MF.

By comparing the new membrane (Fig. 4a) with the fouled membranes (Fig. 4b and c), it could be found that the internal membrane fouling was even formed on the supporting 
material under the active filtration layers of the membranes. The channels on the supporting layer became narrow and obscured near the membrane surface. Compared with fouled membrane of CF-MF, it can be seen clearly that the portion of the internal foulants deposited on the fouled membrane of MEF-MF was slighter. The cake layer on membrane surface of MEF-MF was also thinner than that of CF-MF. The results of microscopic observation demonstrated that the internal fouling induced by deposition or blockage in membrane pores of CF-MF was much more serious than that of MEF-MF, which compared favorably with the results of the membrane flux decline.

\section{MEF for mitigating membrane fouling}

As shown in Fig. 3 and Fig. 4, the MEF pretreatment could mitigate membrane fouling more effectively than flocculation pretreatment. When comparing virgin membrane and two fouled membrane that ran in CF-MF and MEF-MF, the mechanisms of membrane fouling mitigation were explained based on microcosmic morphology.

\section{Floc characterization}

The ordinary explanation for membrane fouling mitigation is the increase in particle size as a result of the flocculation process [26], which prevents internal fouling and produces a cake layer with lower hydraulic resistance. Some studies reported that formation of loose, porous flocs and reduction of small colloidal particles in longer flocculation time led to higher flux.

Fig. 5 depicts the images of flocs formed in CF-MF and MEF-MF with coagulants of $20 \mathrm{mg} / \mathrm{L}$, respectively. The flocs in MEF-MF were significantly larger and denser contrast to that of CF-MF. The results indicated that a lot of ferromagnetic seeds could evenly spread in Fe-flocs.

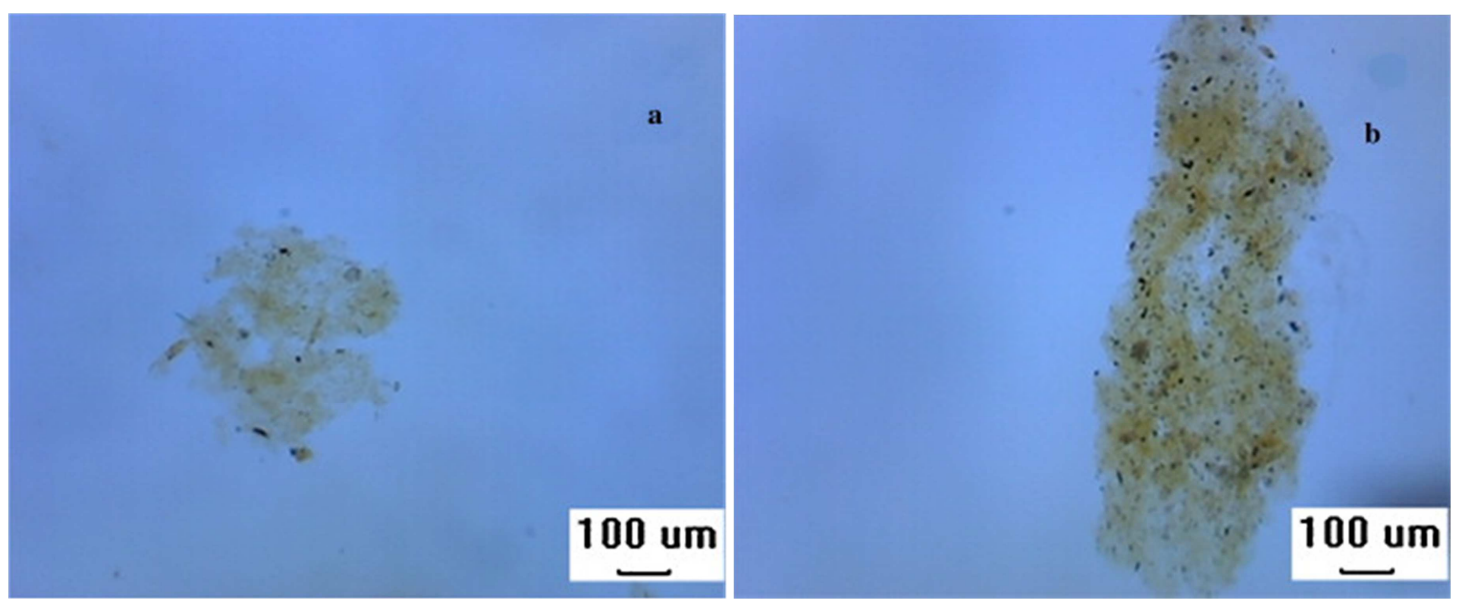


Fig. 5. Images of flocs in different pretreatments process: (a) coagulation/flocculation, (b) MEF. The dosage of coagulant in each pretreatments process was $20 \mathrm{mg} / \mathrm{L}$.

Fig. 6 shows that the average sizes of flocs for both MEF and coagulation/flocculation. It was obviously that the average particle size of flocs in MEF was larger than that of flocs in CFMF at coagulant dosage of $20 \mathrm{mg} / \mathrm{L}$, which verified that flocs spread with ferromagnetic seeds could adsorb more foulants to form larger particle size. As a result, it enhanced back transport from membrane surface to bulk flow and decreased particle deposition on membrane surface [27] and [28].

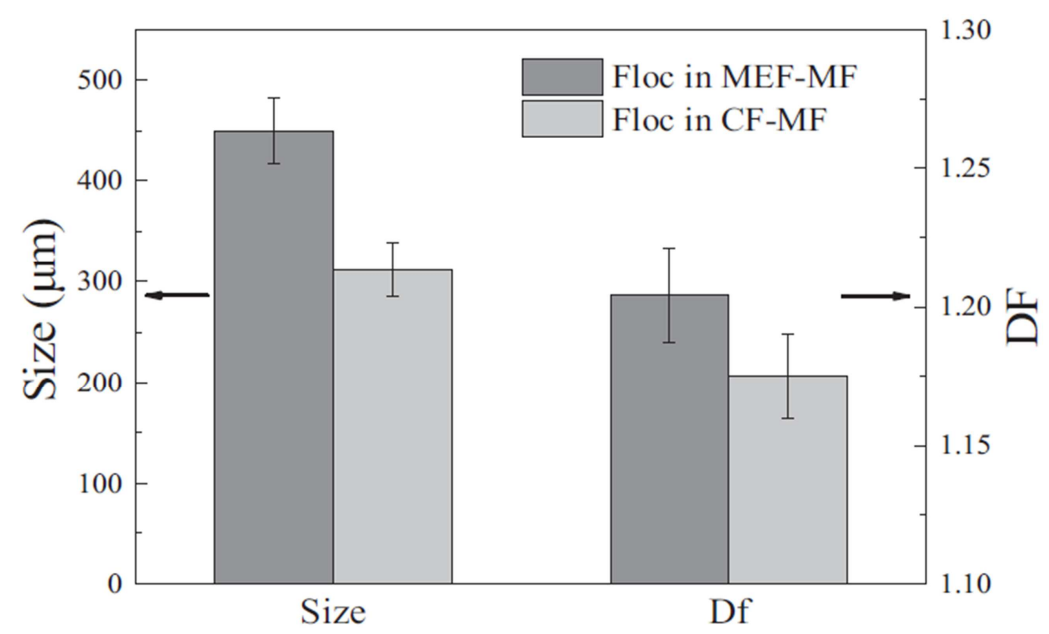

Fig. 6. Flocs parameters of different dosage coagulant: (a) the average diameter (b) fractal dimension. The flocs were collected from the whole process of MEF-MF and CF-MF for every hour in $6 \mathrm{~h}$, respectively. The data points represent the average value of duplicate experiments.

In the fractal concept, the most important and powerful quantitative parameter is fractal dimension $\left(D_{f}\right)$ [29] which indicates the space filling capacity [30], that is, the compactness of flocs. Larger fractal dimensions signify more compact flocs, which are usually preferred in most situations in water treatment to yield lower sludge volumes and easier sedimentation. The analysis of fractal dimension provided important information of flocs since its value relied on the adding of ferromagnetic seeds. As shown in Fig. 6, the fractal dimension of flocs in MEF-MF are larger than that of ordinary flocs, which means the flocs are more stable and easier sedimentation. The existence of ferromagnetic seeds increased the cohesion between the small flocs in which way the flocs with larger fractal dimension were formed.

Flocculation and the characteristic of flocs could also change the molecular weight distribution (MWD) in feeding water. As is shown in Fig. 7, the investigated MEF at optimal 
conditions was more efficient for the reduction of middle molecular weight (MW) organic structures, i.e. humic-like substances (band 2) [31], than flocculation (20 mg/L coagulant). Additionally, high MW structures (band 1) attributed to biopolymers and originating from microbial origin [32] were also significantly reduced by MEF treatment. However, these structures were only partly removed by coagulation/flocculation treatment as is showed in GPC chromatograms.

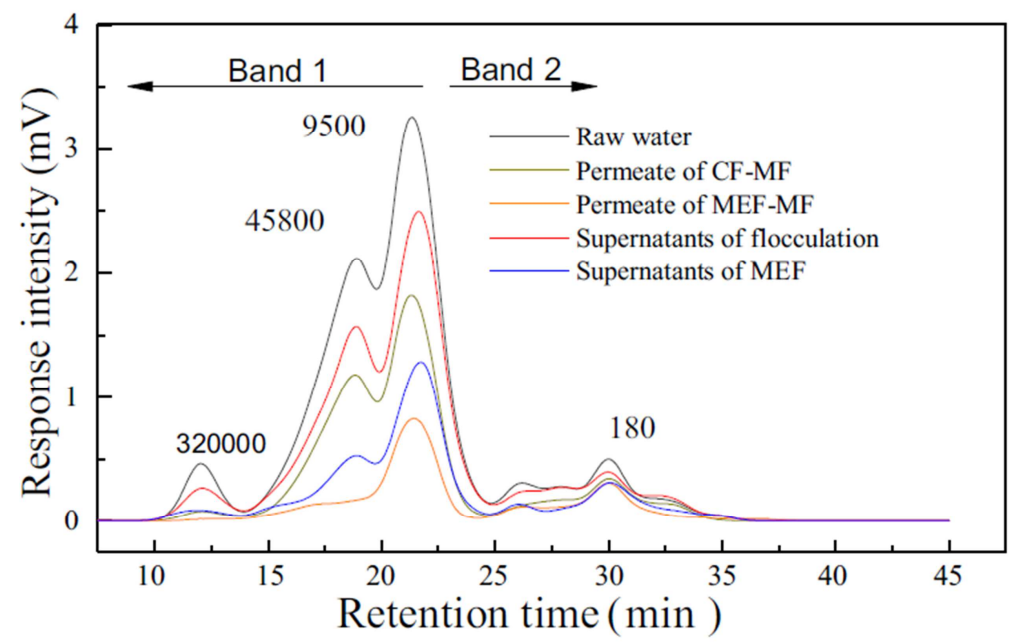

Fig. 7. The molecular weight distribution of foulants in raw water and permeates of CF-MF and MEF-MF, respectively.

As Humbert observed [32], high MW dissolved organic matter (DOM) contributed to a minor part of both DOC and potential difference signals in surface waters. However, such minority substances were shown to contribute to a large extent to the reversible fouling of membrane operated in dead-end filtration mode. It was also demonstrated in Fig. 3 that no significant reduction of membrane flux was observed after ferromagnetic seeds flocculation treatment.

\section{Cake layer analysis}

Fig. 8 shows the light microscope images of fouled membranes of MEF-MF and CF-MF. On the surfaces of fouled membranes there are noticeable cake layers with different structures. The cake layer of MEF-MF is porous and it also appears to be loose and littered with ferromagnetic seeds (Fig. 8a). The cake layer on the membrane surface of CF-MF is relatively less porous, more compact and more equally distributed (Fig. 8b). This is consistent with the findings of the SEM images of the cross-section of membrane, namely that the cake formed with flocs which were mixed with ferromagnetic seeds on membrane surface is slightly more porous. 
Due to the adding of ferromagnetic seeds, the structure of flocs turned relatively larger. Combining above discussion with the fact that all of the experimental conditions were identical except for the existence or absence ferromagnetic seeds, it is suggested that the structure of the cake layer may be attributed to the interaction between ferromagnetic seeds and flocs in the filtration stage.

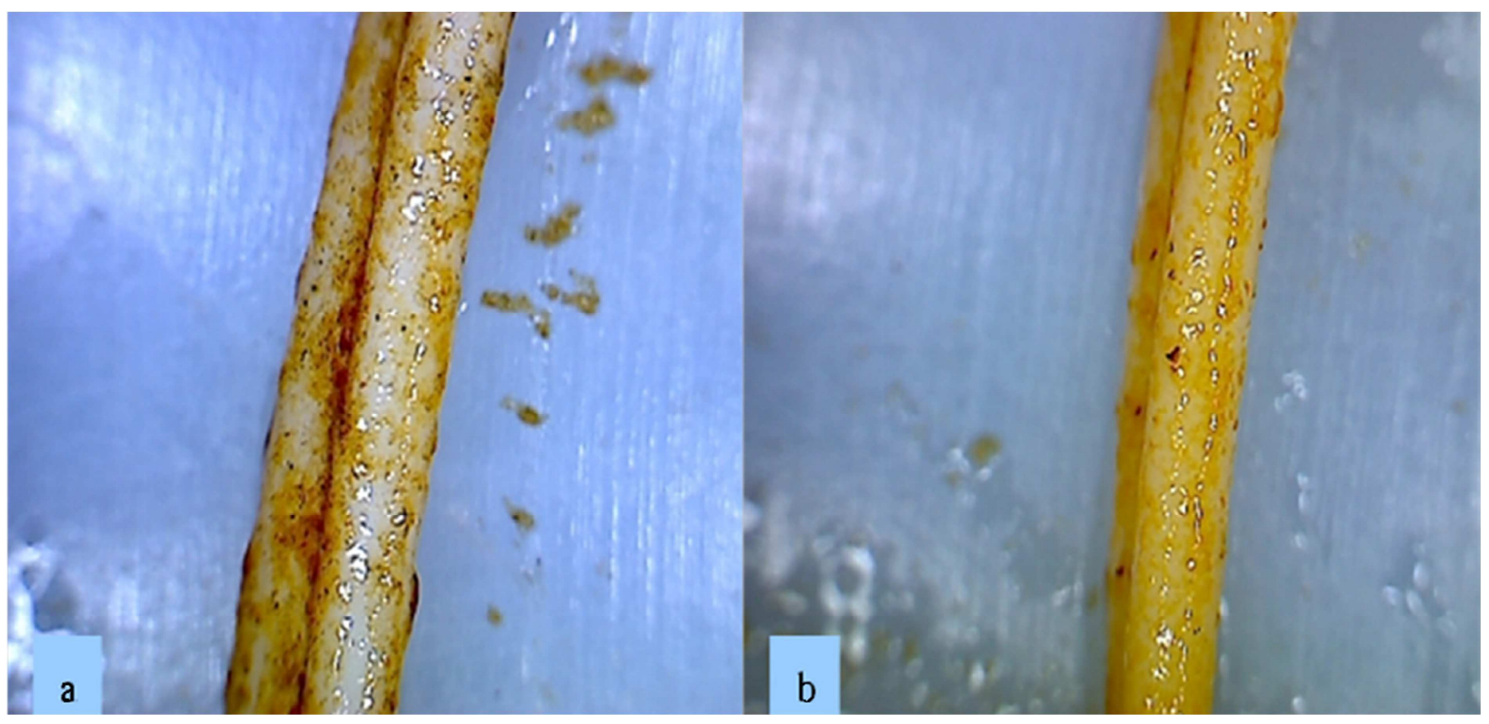

Fig. 8. The pictures of fouled membrane under light microscope: (a) membrane of MEF-MF, (b) membrane of coagulation MF after uninterrupted filtration for $6 \mathrm{~h}$.

According to the Carman-Kozeny theory [33], large flocs can decrease filtration resistance of cake layer and alleviate membrane fouling. When mixture filtrated thought membrane, tiny flocs were intercepted on the membrane surface to form cake layer that easily blocked the membrane pores. Cake layer with no support between flocs formed a dense structure. With the thickening and compacting of cake layer, the porosity reduced, and flux declined rapidly so that a gel layer was easy to form. The enhanced magnetic flocculation could form larger size flocculent body, reduce the number of small molecules particles in the reactor, and the time of small particles clogging the membrane pores in the initial stage of membrane filtration, and promoted the formation of cake layer. Moreover, as the support of ferromagnetic seeds and the interaction between magnetic flocs and membrane surface, the porosity of cake layer and the water permeability were increased to mitigate the membrane fouling.

With membrane filtration period going on, larger colloid particles were absorbed and intercepted in the porous cake layer. The accumulation of pollutants could cause the change of membrane surface zeta potential. The surface $\mathrm{ZP}$ is potential at the electro-kinetic slipping 
plane between the membrane surface and solution when relative motion occurs between them [34] and [35]. It has often been used in membranes to infer the charge of surface and pores. The surface $\mathrm{ZP}$ is an important property that affected membrane fouling.

As shown in Table 1, ZP of raw water used in the experiments is $-30.5 \pm 0.97 \mathrm{mV}$ which is mainly contributed by the biopolymers. The $\mathrm{ZP}$ of the virgin membrane at neutral $\mathrm{pH}$ was $5.8 \pm 1.25 \mathrm{mV}$. Membrane surface ZP after being fouled in each flocculation process was performed using the same membrane coupons. As is shown in Fig. 9 colloid foulants have caused notable changes in membrane surface $\mathrm{ZP}$, manifesting significant deposition on membrane surface. The surface ZPs of fouled membranes in CF-MF and MEF-MF processes decreased to $-32.4 \mathrm{mV}$ and $-19.1 \mathrm{mV}$, respectively. Deposition of the negatively charged colloid led to an increase in the magnitude of the negative membrane surface ZP. It is obviously that ferromagnetic seeds mixed coagulant has a better effect on neutralizing and removing the negative colloid in the flocculation pretreatment before membrane filtration.

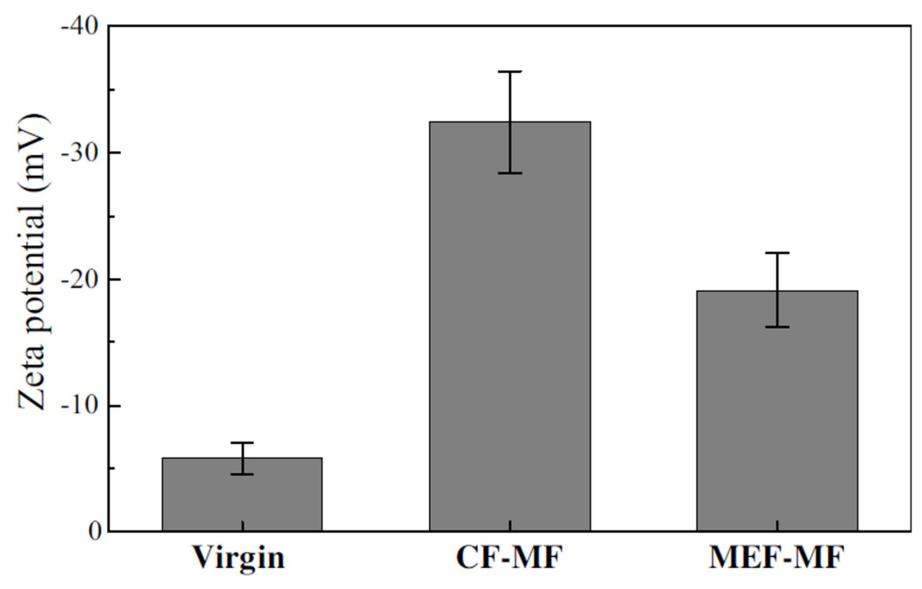

Fig. 9. The surface zeta potential of virgin membrane and fouled membranes. The fouled membranes were collected from CF-MF and MEF-MF processes, respectively.

In another hand, magnetic filter cake layer can intercept macromolecular substance effectively. By contrasting the MWD of permeate and supernatants in Fig. 7, it is obvious that membrane filtration could intercept part of middle MW for both CF-MF and MEF-MF. However, high MW (protein-like substances) and small molecules detected in the supernatants and permeate of CF-MF implied that protein-like substances existed continuously during the process of the membrane filtration, while they are minority in supernatants and permeate of MEF-MF.

Membrane foulant analysis 
The FTIR spectra of virgin and fouled membranes are illustrated in Fig. 10. The specific constituents of organic matter causing membrane fouling cannot be confirmed by FTIR analysis because of their complexity, but it is still a very useful method to identify the functional group compositions. The most decisive absorption of PVDF is the strong peak at $1400 \mathrm{~cm}^{-1}$ that pertains to $\mathrm{C} \_$F stretching. It also has a broad peak at $3289 \mathrm{~cm}^{-1}$ for $\_-O H$, and at $900-690 \mathrm{~cm}^{-1}$ for $\mathrm{C} \_$H. When substances were deposited in membrane pores and on membrane surface, the aforementioned functional groups of PVDF were masked and their peak intensities diminished.

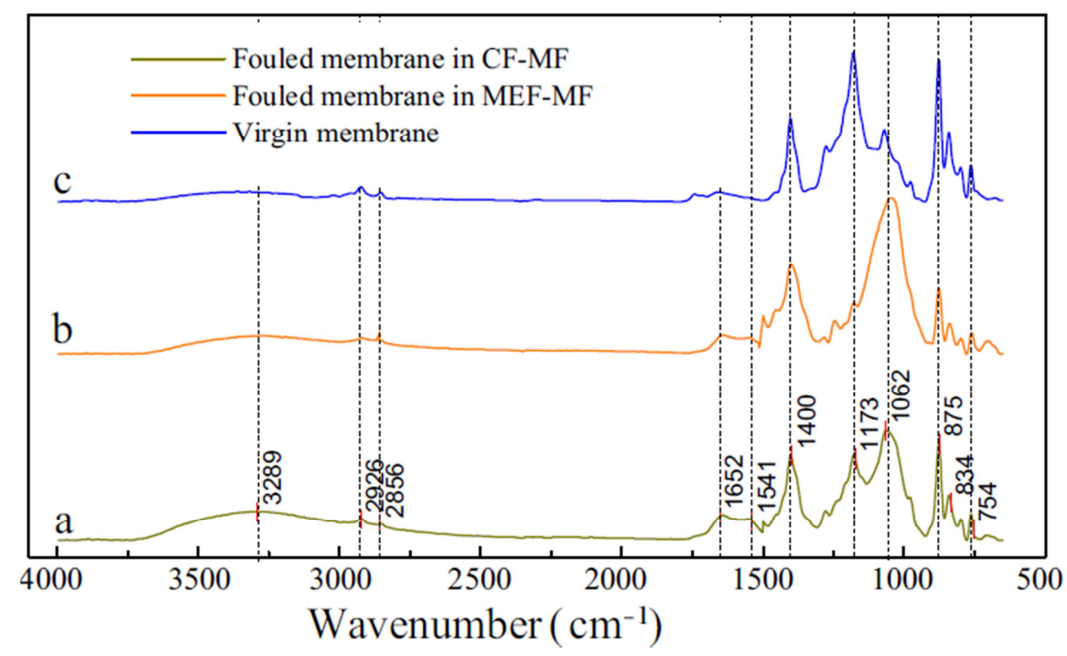

Fig. 10. The FTIR spectra of (a) virgin, (b) fouled membrane in MEF-MF and(c) fouled membrane in CF-MF.

The fouled membranes of CF-MF and MEF-MF have weak absorption at $2921 \mathrm{~cm}^{-1}$ and $2856 \mathrm{~cm}^{-1}$ for $\mathrm{C} \_$H stretching vibration and $1062 \mathrm{~cm}^{-1}$ for $\mathrm{C}_{-} \mathrm{O}$ noting the presence of polysaccharides. Absorptions that appears at $1541 \mathrm{~cm}^{-1}$ and $1652 \mathrm{~cm}^{-1}$ can refer to amide II $\left(\mathrm{N} \_\mathrm{H}\right.$ bending and $\mathrm{C}-\mathrm{N}$ stretching vibrations) and amide $\mathrm{I}(\mathrm{C}=\mathrm{O}$ stretching and other secondary structure vibrations), respectively. The existed polysaccharides and protein-like were the main component of biopolymers.

Relatively strong FTIR intensities of polysaccharide and protein-like functional groups found in the fouled membrane compared to the virgin membrane indicated the main foulants dominating the zeta potential reduction and membrane fouling. However, these protein peaks are also in close proximity to well-known absorption of humic acids, particularly the aromatic $\left(1620 \mathrm{~cm}^{-1}\right)$ and carboxylate $\left(1600-1400 \mathrm{~cm}^{-1}\right)$ groups [36], which raises the possibility of peak overlapping. And humic acids might be present on the fouled membrane surface as well. 
Fluorescence spectroscopy has been widely used to investigate the chemical properties and source of DOM in natural waters [37], and especially three-dimensional excitation emission matrix (EEM) fluorescence spectroscopy has been successfully used to probe the chemical structure of DOM because they can distinguish among different classes of DOM of different origins [38], [39] and [40]. It has been investigated as a monitoring tool for a range of application including water quality and pollution monitoring in rivers. In terms of the subtraction spectrum of fluorescence EEMs between the raw water and permeate, the removal effect of organic matters can be visually and qualitatively reflected in this study.

The EEM spectrum (Fig. 11) qualitatively indicates the DOM composition in raw water. Four peaks have been detected. A peak is present at excitation $\lambda_{\mathrm{EX}}=220-240$ and emission $\lambda_{\mathrm{EM}}=330-380$ where is related to protein-like substances. The second and third peak are observed at $\lambda_{\mathrm{EX}}=240-250$ and $\lambda_{\mathrm{EM}}=420-450$ and $\lambda_{\mathrm{EX}}=300-320$ and $\lambda_{\mathrm{EM}}=390$ 410 where are related to humic and fulvic-like material. The forth fluorescence center is observed at $\lambda_{\mathrm{EX}}=260-270$ and $\lambda_{\mathrm{EM}}=300-320$ where is related to microbial-derived organic matter. These peaks have been widely identified in previous studies.

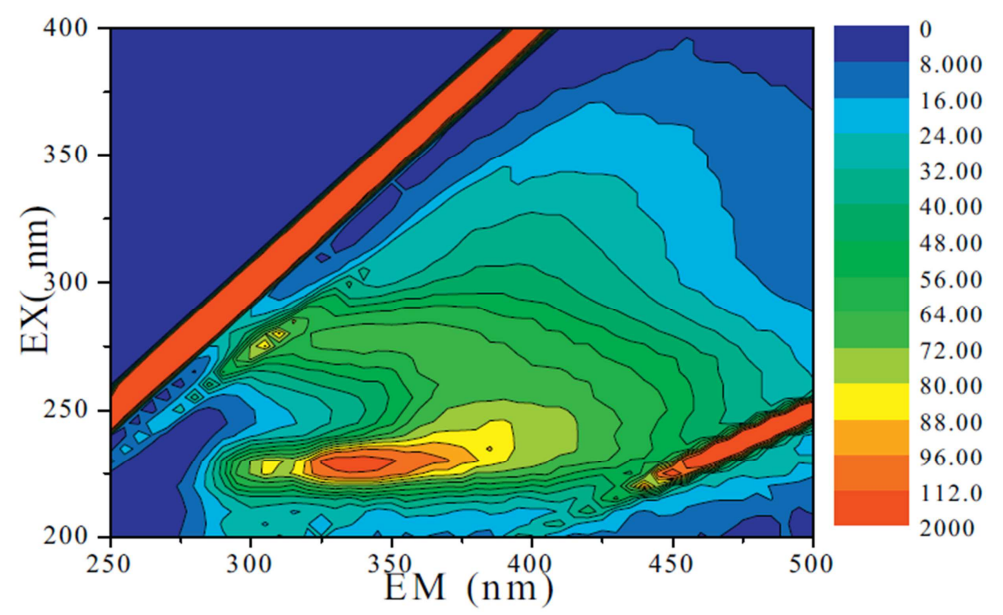

Fig. 11. Fluorescence EEM of raw water.

The full fluorescence EEM spectrums of the supernatants of MEF and flocculation as well as each subtraction spectrums compared to raw water are shown in Fig. 12. These spectrums indicate that protein-like and microbial-derived substances were mostly removed in MEF-MF compared to humic-like compounds obviously. However, these protein-like and microbialderived substances only partly removed in CF-MF. Substances resulting from microbial activity may be high molecular weighted and more insoluble than humic-like substances and therefore less refractory to flocculation process. By compare Fig. $12 \mathrm{~b}$ and $\mathrm{d}$, it is clear that 
the MEF-MF process could get rid of protein-like compounds from mixture than that of CFMF. However, both of CF-MF and MEF-MF have unattractive effect on removal of humic and fulvic-like material present around $\lambda_{\mathrm{EX}} / \lambda_{\mathrm{EM}}=240-250 / 420-450$ in the EEM spectrums.
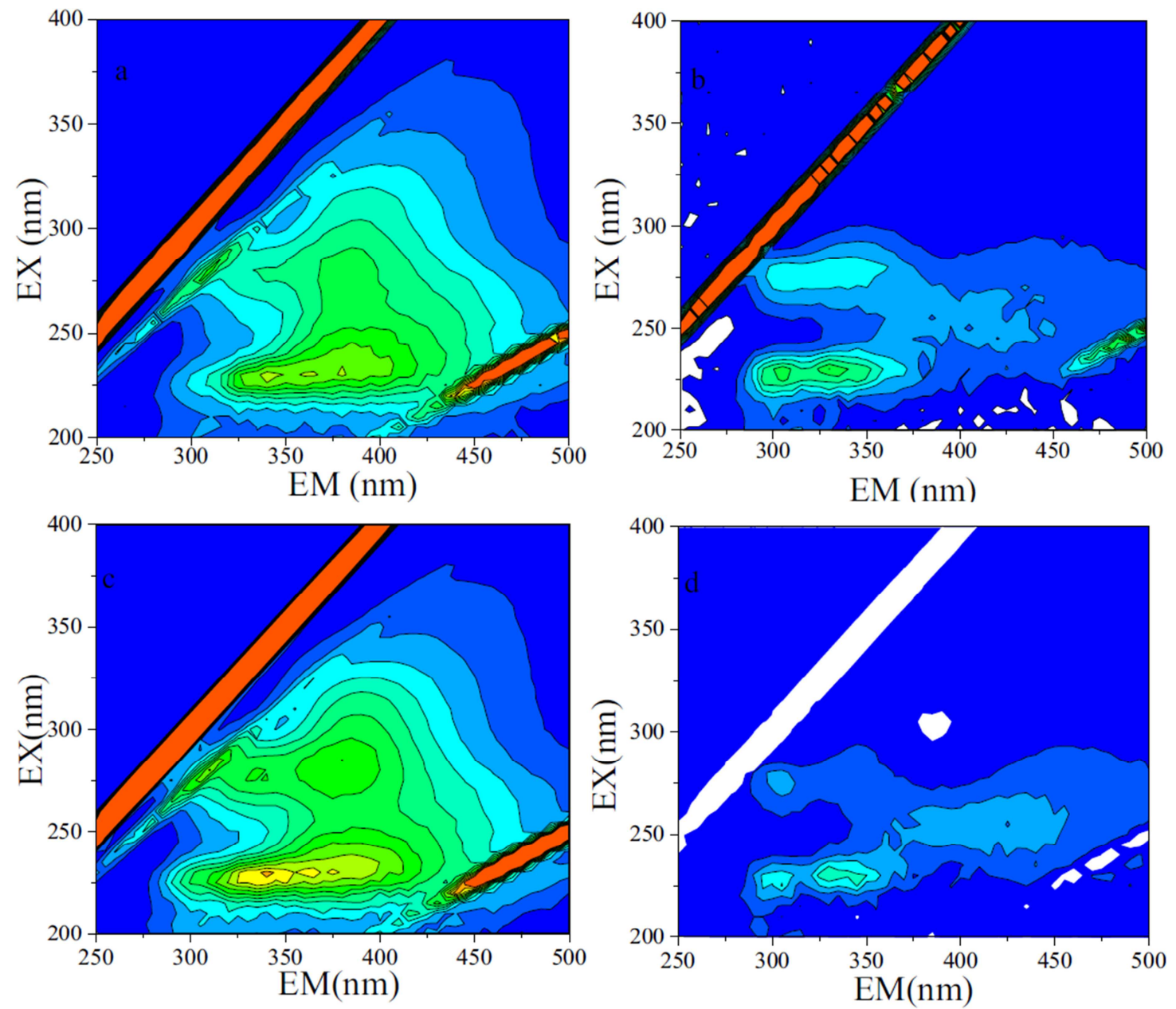

Fig. 12. The full fluorescence EEM spectrums and the subtraction spectrums of raw water and permeate (a) the permeate of MEF-MF, (b) subtraction spectrum of MEF, (c) the permeate of CF-MF, (d) subtraction spectrum of CF-MF.

It is found that biopolymers especially protein-like possibly accumulated in greater proportion on fouled membrane surface of CF-MF. By contrast, biopolymers are effectively removed before filtration in MEF pre-treatment, in which way their accumulation on the membrane surface was reduced. This demonstrates that biopolymers are the major species accountable for the membrane fouling potential in CF-MF process.

In general, MEF was effective to mitigate membrane fouling as pretreatment for drinking water ultrafiltration treatment. With the aspect of flocs, MEF could improve the $D f$ and size 
of floc with ferromagnetic seeds. Porous cake layer with flocs of large size and $D f$ was helpful for alleviating the decline rate of membrane flux. As for the foulants, analyses by GPC and EEM have proved that MEF pretreatment was more efficient for the reduction of low and mid-MW organic structures, i.e. humic-like material, than flocculation pretreatment. High MW structures of biopolymers were also significantly reduced by MEF pretreatment. The zeta potential analysis of the fouled membranes also verified the accumulation of negative charged colloidal particles on the membrane surface of MEF-MF was less than those of CFMF.

\section{The model development of MEF-MF}

The results of this work indicate that ferromagnetic seeds could play a significant role in flocculation for mitigating membrane fouling. A reasonable ferromagnetic seeds flocculation mechanism has been described schematically in Fig. 14. Fe species clusters coated with hydration layer are formed when ferromagnetic seeds were added. The hydroxyl $(-\mathrm{OH})$ in hydration layer can form chemical bonds with Fe ions in the FC under a week magnetic field.

The formed clusters would increase the proportion of iron around the ferromagnetic seeds, which has a great potential to enhance charge neutralization, enmeshment and adsorption when aggregated with the pollutants. In addition, magnetism is a unique physical property that independently contributes to flocculation by influencing the physical properties of contaminants in water. The flocs produced by the coagulants exhibit different characteristics as shown in Fig. 5.

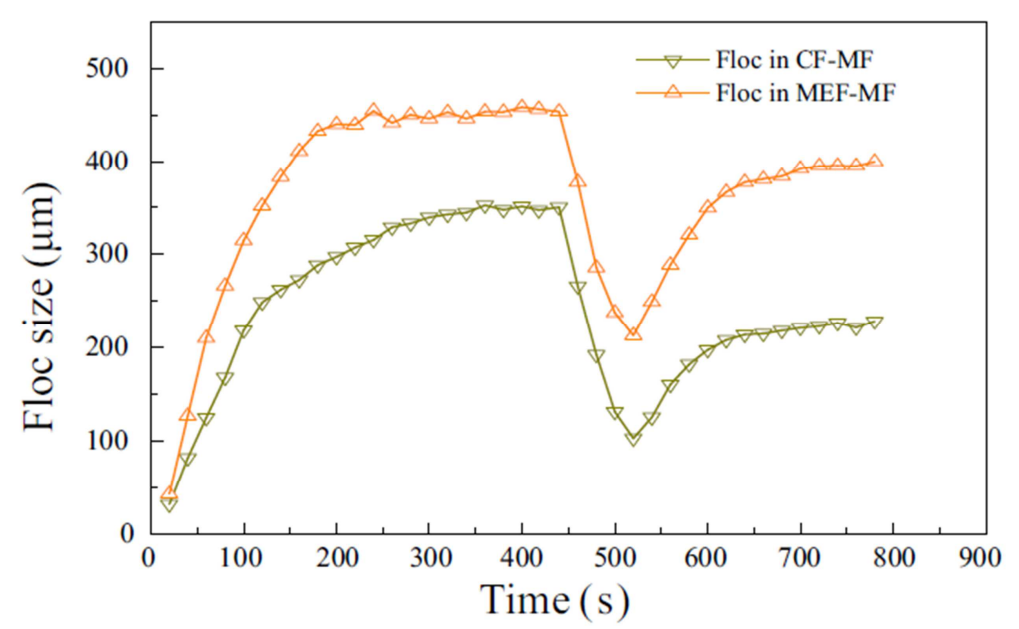

Fig. 13. The comparison of the floc formation kinetics curves. The dosage of FC in CF-MF was $20 \mathrm{mg} / \mathrm{L}$, and the dosage of ferromagnetic seeds mixed FC in MEF-MF was also 
$20 \mathrm{mg} / \mathrm{L}$. Both jar-tests were conducted as follows: a slow stir phase at $30 \mathrm{rpm}$ for $7 \mathrm{~min}$, followed by a breakage phase at $200 \mathrm{rpm}$ for $1.5 \mathrm{~min}$. After the breakage phase, the slow stir at $30 \mathrm{rpm}$ was reintroduced for a further $5 \mathrm{~min}$.

And as illustrated in Fig. 13, the flocs formed by ferromagnetic seeds mixed FC were larger than that of FC. As stated previously, ferromagnetic seeds exhibit a preference to form $\mathrm{Fe}$ species clusters. These clusters enhance the bridge and adsorption effects, leading to larger floc size. Moreover, the recovery factors of the flocs show marked differences. The floc recovery factor of FC $(20 \mathrm{mg} / \mathrm{L})$ was very low $(56 \%)$, while that of ferromagnetic seeds mixed FC was high (89\%), which implied that the connections between the clusters and organic pollutants were unique basing on chemical bonds rather than on physical ones. Flocs formed by FC showed a different situation. They were weaker and comparable small. Aggregation of nanoparticles was easy, and thus the dosage of FC coagulant reduced.

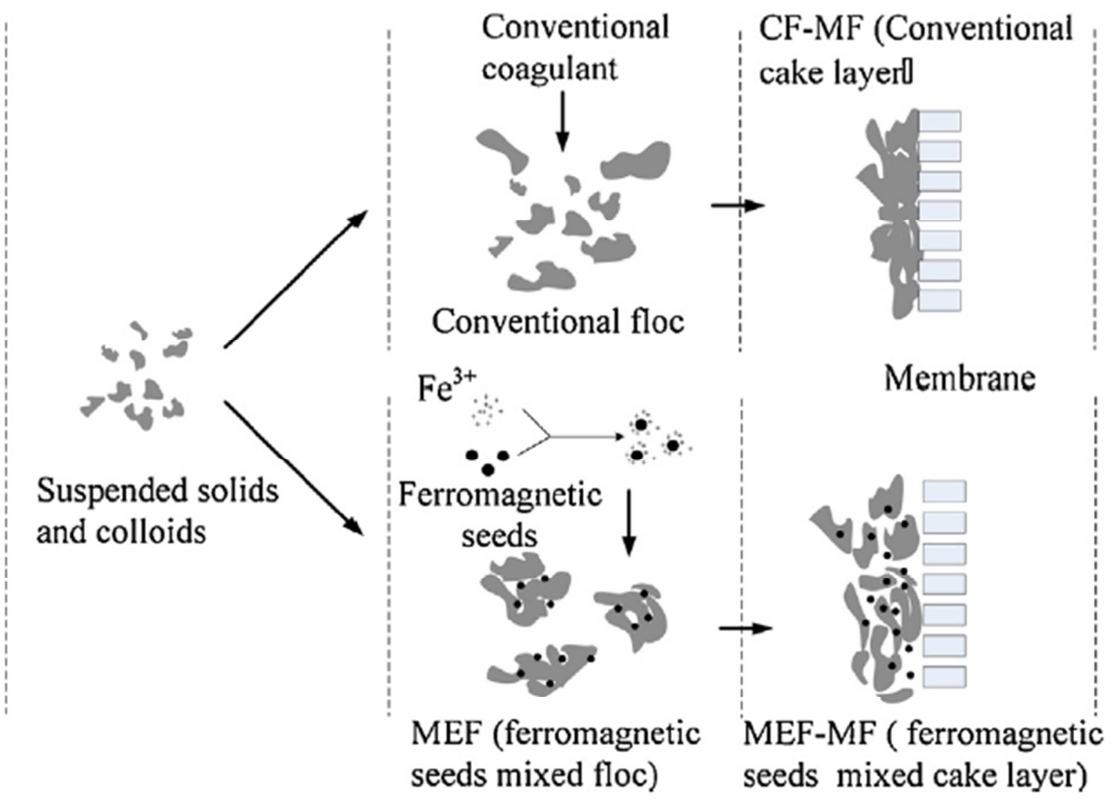

Fig. 14. The schematic diagram of MEF-MF and CF-MF for coagulation mechanism.

Additionally, the formed flocs were considerably porous and fractal. The excess colloids (i.e. humic-like material and biopolymers) could penetrate into flocs easily. The embodiment of those colloids would increase the $D f$ of the flocs. The particles were entrapped in cake layer formed by the denser flocs to protect membrane from small particles blocking membrane pore so as to mitigate the membrane permanent fouling. Additionally, cake layer formed by magnetic flocs was more porous than by general flocs, which made flux permeate through cake layer more easily and enhancd the performance of membrane process. 


\section{Conclusions}

Compared to CF-MF, MEF-MF had a palpable superiority on mitigating membrane fouling. Higher fractal dimension and larger flocs spread with ferromagnetic seeds were formed in MEF pretreatment. In the membrane filtration process, porous cake layer was constructed with the magnetic flocs depositing on membrane surface gradually, which made permeate flux pass cake layer easier and enhanced the performance of membrane process.

Foulants especially the high MW biopolymers were almost trapped in the ferromagnetic seeds spread flocs and cake layer, preventing clogging membrane pores. Moreover, low and mid-MW organic structures, i.e. humic-like materials were also partially removed by MEF pretreatment. With the accumulation of colloids on membrane surface in CF-MF process, the membrane potential decreased significantly comparing to that in MEF-MF process.

In consideration of the effect of ferromagnetic seeds on mitigating membrane fouling, MEFMF can provide a novel alternative approach for drinking water treatment in the future.

\section{Acknowledgments}

This study is financially supported by the National Natural Science Foundation of China (No.51108314, No.51378349) and China Postdoctoral Science Foundation (2013M541184).

\section{References}

[1] D.H. Kim, H.K. Shon, G. Sharma, J. Cho, J. Ind. Eng. Chem. 17 (2011) 109.

[2] T. Zsirai, P. Buzatu, P. Aerts, S. Judd, Water Res. 46 (2012) 4499.

[3] J.Y. Tian, Z.L. Chen, Y.L. Yang, H. Liang, J. Nan, G.B. Li, Water Res. 44 (2010) 59.

[4] A. Bagga, S. Chellam, D.A. Clifford, J. Membr. Sci. 309 (2008) 82.

[5] N.P. Gamage, S. Chellam, J. Membr. Sci. 379 (2011) 97.

[6] R. Bergamasco, L.C. Konradt-Moraes, M.F. Vieira, M.R. Fagundes-Klen, A.M. Sal-cedo Vieira, Chem. Eng. J. 166 (2011) 483.

[7] W.Z. Yu, N. Graham, H.J. Liu, J.H. Qu, Chem. Eng. J. 234 (2013) 158.

[8] R. Mao, Y. Wang, Y. Zhao, B. Gao, M. Dong, Chem. Eng. J. 225 (2013) 387.

[9] S.J. Lee, K.H. Choo, C.H. Lee, J. Ind. Eng. Chem. 6 (2000) 357.

[10] C.P. Leo, N.H.A. Kamil, M.U.M. Junaidi, S.N.M. Kamal, A.L. Ahmad, Sep. Purif. Technol. 103 (2013) 84. 
[11] D.J. Lee, G.Y. Liao, Y.R. Chang, J.S. Chang, Int. J. Hydrogen Energy 37 (2012) 15643.

[12] Y. Matsui, H. Hasegawa, K. Ohno, T. Matsushita, S. Mima, Y. Kawase, T. Aizawa, Water Res. 43 (2009) 5160.

[13] S. Yiacoumi, D.A. Rountree, C. Tsouris, J. Colloid Interface Sci. 184 (1996) 477.

[14] C. Tsouris, T.C. Scott, J. Colloid Interface Sci. 171 (1995) 319.

[15] J. Svoboda, J. Zofka, J.Colloid Interface Sci. 94 (1983) 37.

[16] Y. Li, J. Wang, Y. Zhao, Z. Luan, Sep. Purif. Technol. 73 (2010) 264.

[17] S. Zakaria, B.H. Ong, T.G.M. van de Ven, Colloids Surf., A: Physicochem. Eng. Aspects 251 (2004) 1.

[18] J. Guo, X. Li, G. Wu, Z. Wang, J. Song, Q. Feng, Adv. Mater. Res. 1867 (2012) 356360.

[19] D. Liu, P. Wang, G. Wei, W. Dong, F. Hui, Environ. Sci. Pollut. Res. 20 (2013) 60.

[20] G.U. Semblante, S.D.R. Tampubolon, S.J. You, Y.F. Lin, T.C. Chang, F.C. Yen, J. Membr. Sci. 435 (2013) 62.

[21] M. Zhang, F. Xiao, X.Z. Xu, D.S. Wang, Water Res. 46 (2012) 127.

[22] S. Salgin, U. Salgin, N. Soyer, Int. J. Electrochem. Sci. 8 (2013) 4073.

[23] M.C. Wilbert, S. Delagah, J. Pellegrino, J. Membr. Sci. 161 (1999) 247.

[24] Y. Adachi, M. Kobayashi, S. Ooi, J. Colloid Interface Sci. 208 (1998) 353.

[25] K. Xiao, X. Wang, X. Huang, T.D. Waite, X. Wen, J. Membr. Sci. 373 (2011) 140.

[26] W.S. Guo, S. Vigneswaran, H.H. Ngo, Desalination 172 (2005) 53.

[27] S. Kim, H. Park, J. Environ. Eng.-ASCE 128 (2002) 335.

[28] S. Kim, H. Park, J. Environ. Eng.-ASCE 131 (2005) 865.

[29] Y. Zhou, G.V. Franks, Langmuir 22 (2006) 6775.

[30] D.N. Thomas, S.J. Judd, N. Fawcett, Water Res. 33 (1999) 1579.

[31] H. Humbert, H. Gallard, J.P. Croue, Water Res. 46 (2012) 1093.

[32] H. Humbert, H. Gallard, V. Jacquemet, J.-P. Croue, Water Res. 41 (2007) 3803.

[33] P.K. Park, C.H. Lee, S. Lee, Environ. Sci. Technol. 40 (2006) 2699.

[34] Q. Yun-Ren, Q. Jing, J. Membr. Sci. 425-426 (2013) 71.

[35] R. Blank, K.H. Muth, S. Proske-Gerhards, E. Staude, Colloids Surf., A: Physicochem. Eng. Aspects 140 (1998) 3.

[36] A.W. Zularisam, A.F. Ismail, M.R. Salim, M. Sakinah, O. Hiroaki, J. Membr. Sci. 299 (2007) 97.

[37] S. Li, W. Sun, Chem. Eng. J. 237 (2014) 101.

[38] S. Peldszus, C. Halle, R.H. Peiris, M. Hamouda, X. Jin, R.L. Legge, H. Budman, C. Moresoli, P.M. Huck, Water Res. 45 (2011) 5161.

[39] G.A. Baddi, B. Antizar-Ladislao, A. Alcuta, L. Mazeas, T. Li, C. Duquennoi, E. Redon, T. Bouchez, Environ. Eng. Sci. 30 (2013) 232. 
[40] E.M. Carstea, A. Baker, M. Bieroza, D. Reynolds, Water Res. 44 (2010) 5356. 